\title{
Levantamento fitossociológico de plantas daninhas em pastagens degradadas do Médio Vale do Rio Doce, Minas Gerais ${ }^{1}$
}

\author{
Evander Alves Ferreira ${ }^{2}$, Anselmo Grossi Fernandez ${ }^{3}$, Celiomar Pereira de Souza ${ }^{4}$, Marcia Aparecida Felipe ${ }^{5}$, \\ José Barbosa dos Santos ${ }^{6}$, Daniel Valadão Silva ${ }^{7}$, Fernanda Aparecida Rodrigues Guimarães ${ }^{8}$
}

http://dx.doi.org/10.1590/0034-737X201461040008

\section{RESUMO}

O levantamento fitossociológico é uma ferramenta importante, na análise do impacto que os sistemas de manejo e as práticas agrícolas exercem sobre a dinâmica de crescimento e ocupação das plantas daninhas no agroecossistema. Por essa razão, esta pesquisa teve como objetivo realizar a caracterização fitossociológica de plantas daninhas, em oito áreas de pastagens degradadas, de cinco municípios do Médio Vale do Rio Doce, em Minas Gerais. No total foram identificadas 68 espécies, pertencentes a 18 famílias, sendo que o gênero Sida ocorreu em todas as áreas avaliadas. De maneira geral, a Uroclhoa decumbens, a Sida glaziovii e a Sida carpinifolia foram as espécies encontradas com maior frequência. Todavia, constatou-se grande diversidade de plantas daninhas nas áreas avaliadas, sendo a maior riqueza observada no município de Galiléia e, a menor, em Governador Valadares. As áreas estudadas apresentaram baixa similaridade entre si, sendo, portanto, indicado que o manejo de plantas daninhas seja específico para cada situação.

Palavras-chave: pecuária, fitossociologia, recuperação de áreas degradadas.

\section{ABSTRACT \\ Phytosociological survey of weeds in degraded pasture in the Middle Rio Doce Valley, Minas Gerais State, Brazil}

The phytosociological study is an important tool in analyzing the impact that the management systems and agricultural practices have on the dynamics of weed growth and occupation of agroecosystems. In this sense, this research aims to perform the phytosociological survey of weed in eight areas of degraded pasture in five municipalities of the Middle Rio Doce Valley Minas Gerais. A total of 68 species belonging to 18 families were identified. The genus Sida occurred in all assessed areas. In general, Uroclhoa decumbens, Sida glaziovii and Sida carpinifolia were the most frequently found species. However, a great diversity of weeds was found among the assessed areas, with the highest richness found in the village of Galiléia and the lowest in Governador Valadares. The areas studied showed low similarity to each other, indicating that weed management must be specific to each situation.

Key words: livestock, phytossociology, rehabilitation of degraded areas.

\footnotetext{
Enviado para publicação em 18/10/2012 e aprovado em 02/12/2013.

${ }^{1}$ Agências financiadoras: Capes e FAPEMIG.

${ }^{2}$ Engenheiro-Agrônomo, Doutor. Universidade Federal dos Vales do Jequitinhonha e Mucuri, Rodovia MGT 367, Km 583, 5000, 39100-000, Alto da Jacuba, Diamantina, Minas Gerais, Brasil. evanderalves@gmail.com

${ }^{3}$ Engenheiro-Agrônomo. Universidade Vale do Rio Doce, Rua Moreira Sales, 850, Vila Bretas, 35030-390, Governador Valadares, Minas Gerais, Brasil. agronomo_33@ yahoo.com.br ${ }^{4}$ Engenheiro-Agrônomo. Universidade Vale do Rio Doce, Rua Moreira Sales, 850, Vila Bretas, 35030-390, Governador Valadares, Minas Gerais, Brasil. celiomaragro@ yahoo.com.br ${ }^{5}$ Engenheira-Agrônoma. Universidade Vale do Rio Doce, Rua Moreira Sales, 850, Vila Bretas, 35030-390, Governador Valadares, Minas Gerais, Brasil. maapfel@yahoo.com.br ${ }^{6}$ Engenheiro-Agrônomo, Doutor. Docente da Universidade Federal dos Vales do Jequitinhonha e Mucuri, Rodovia MGT 367, Km 583, 5000, Alto da Jacuba, Diamantina, Minas Gerais, Brasil. jbarbosasantos@yahoo.com.br

${ }^{7}$ Engenheiro-Agrônomo, Mestre. Departamento de Fitotecnia, Universidade Federal de Viçosa, Campus Viçosa, Avenida Peter Henry Rolphs, s/n, 36.570-000, Viçosa, Minas Gerais, Brasil. danielvaladaos@yahoo.com (autor para correspondência).

${ }^{8}$ Engenheira- Agrônoma. Departamento de Fitotecnia, Universidade Federal de Viçosa, Campus Viçosa, Avenida Peter Henry Rolfs, s/n, 36.570-000, Viçosa, Minas Gerais, Brasil. fernanda.guimaraes@ufv.br
} 


\section{INTRODUÇÃO}

A forma e o grau de intervenção humana na ocupação do solo, na Região do Médio Vale do Rio Doce, Minas Gerais, iniciando-se com a exploração da madeira e culminando com a atividade de pecuária extensiva, aliados à fragilidade do ambiente, determinaram um padrão de uso não sustentável. Como consequência, tem-se observado elevada degradação desta região, o que pode ocasionar redução no valor das terras e atrasar a idade de abate dos animais (Curtinhas et al., 2010; Peron \& Evangelista, 2004).

Os solos do Vale do Rio Doce, em Minas Gerais, foram utilizados, em sua maioria, com pastagens plantadas e uso constante do fogo como técnica de renovação (Fávero et $a l ., 2008)$. Essa prática resultou em perdas de nutrientes por volatilização, escorrimento superficial e lixiviação, além de expor o solo ao impacto direto das gotas das chuvas, acelerando os processos erosivos (Kauffman et al., 1998; Ellingson et al., 2000; Fávero et al., 2008).

Um dos problemas resultantes da degradação causada pelo manejo inadequado das pastagens é a infestação de plantas daninhas, que, por sua capacidade de interferência, reduzem a produtividade das forrageiras. Ao competir pelos fatores de crescimento, as plantas daninhas promovem queda da capacidade de suporte da pastagem, aumentam o tempo de formação e de recuperação do pasto, podem causar ferimentos e, ou, intoxicação aos animais e comprometem a estética da propriedade (Silva et al., 2002; Silva et al., 2013).

A realização de estudos fitossociológicos permite avaliar a composição das espécies da vegetação de cobertura, obtendo-se frequência, frequência relativa, densidade, densidade relativa, abundância, abundância relativa e índice de importância relativa, sendo importante ferramenta utilizada na inferência sobre a comunidade em questão (Gomes et al., 2010). Post (1988) estabeleceu duas causas para a desuniforme distribuição das plantas daninhas no campo: a primeira delas refere-se ao processo de dispersão, em si; e, a segunda, à variabilidade espacial das condições que regulam a germinação, o que pode causar variações nas densidades populacionais, mesmo quando as sementes são uniformemente distribuídas na área. Se as condições ecológicas tendem a favorecer a germinação de uma determinada semente, devem também favorecer a germinação de outras próximas a ela, condicionando o aparecimento de reboleiras.

Segundo Pitelli (2000 a, b), os índices fitossociológicos são importantes para se analisar o impacto que os sistemas de manejo e as práticas agrícolas exercem sobre a dinâmica de crescimento e ocupação de comunidades infestantes em agroecossistemas. Levando em conta as premissas do manejo sustentável, as quais incluem a manutenção de condições mínimas à perpetuação dos biomas e capacidade de regeneração de ambientes degradados, estudos fitossociológicos, em áreas de pastagens, são fundamentais para o correto direcionamento de práticas adequadas para correção e manutenção da capacidade de suporte. Nesse sentido, considerando-se a carência de informações na região, estudos que evidenciem a infestação e a caracterização de plantas daninhas nas áreas de pastagens são imprescindíveis, com o objetivo de adotar medidas que proporcionem adequada avaliação da estrutura da vegetação, visando ao correto manejo durante a implementação de etapas como reforma ou introdução de sistemas de integração.

Por esta razão, objetivou-se, com este trabalho, realizar a caracterização fitossociológica de plantas daninhas em áreas de pastagens representativas da Região do Médio Vale do Rio Doce, em Minas Gerais.

\section{MATERIAL E MÉTODOS}

Os levantamentos foram realizados em oito áreas, de cinco municípios do Médio Vale do Rio Doce (Tabela 1), com histórico de pastagens degradadas pela atividade pecuária, situadas no terço médio do terreno, com solo de textura argilosa e predomínio de pasto formado com espécies do gênero Urochloa. O clima da região avaliada é Aw, isto é, com inverno seco e chuvas concentradas no verão, de acordo com a classificação de Köppen-Geiger.

Para o estudo fitossociológico, utilizou-se o método do quadrado inventário, aplicado por meio de um quadrado de $0,5 \mathrm{~m}$ de lado, lançado ao acaso, em média, 23 vezes em cada área, perfazendo um total de 184 parcelas. A cada lançamento, as espécies encontradas dentro do quadrado foram colhidas, identificadas e cadastradas, sendo obtido o número de indivíduos por espécie.

O levantamento foi realizado durante o segundo semestre de 2007. O sistema de classificação utilizado foi o APG III (Angiosperm Phylogeny Group III). Após a coleta das plantas nas áreas, foram estimadas a frequência (FRE) - que permite avaliar a distribuição das espécies nas parcelas; a densidade (DEN) - quantidade de plantas de cada espécie por unidade de área $\left(\mathrm{m}^{2}\right)$; a abundância

Tabela 1: Caracterização das áreas representativas de pastagens degradadas no Médio Vale do Rio Doce, Minas Gerais, Brasil

\begin{tabular}{ll}
\hline Área & \multicolumn{1}{c}{ Município } \\
\hline 1 & Frei Inocêncio \\
2 & Galiléia \\
3 & São Vitor \\
4 & Engenheiro Caldas - Divino do Traira \\
5 & Governador Valadares - Baguarí-Norte \\
6 & Engenheiro Caldas \\
7 & Governador Valadares - Baguarí-Sul \\
8 & Governador Valadares - Marcos Bretas \\
\hline
\end{tabular}

Rev. Ceres, Viçosa, v. 61, n.4, p. 502-510, jul/ago, 2014 
$(A B N)$ - que informa sobre a concentração das espécies na área; a frequência relativa (FRR), a densidade relativa (DER) e abundância relativa (ABR) - que determinam a relação de cada espécie com outras encontradas na área; e o índice de valor de importância (IVI) - que indica quais espécies são mais importantes dentro da área estudada (Mueller-Dombois \& Ellenberg, 1974).

Ao final, estabeleceu-se a comparação entre as áreas, por meio do índice de similaridade (IS). Para avaliação da similaridade, entre as populações botânicas, nas oito áreas estudadas, foi utilizado o IS - Índice de Similaridade de Sorensen (Sorensen, 1972). O IS varia de 0 a 100, sendo máximo quando todas as espécies são comuns às duas áreas e mínimo quando não existem espécies em comum.

\section{RESULTADOS E DISCUSSÃO}

$\mathrm{Na}$ área 1, Município de Frei Inocêncio, foram identificadas 23 espécies, distribuídas em dez famílias, sendo a Poaceae a mais representativa, com oito, no total, seguida de Malvaceae e Fabaceae, com cinco e três espécies, respectivamente (Tabela 2). Grande número de plantas daninhas é citado como infestante em pastagens e as famílias mais importantes são: Leguminosae, Poaceae, Malvaceae, Myrtaceae, Cyperaceae, Asteraceae, Rubiaceae, Lamiaceae e Apiaceae (Silva \& Dias Filho, 2001; Lara et al., 2003). Todas as espécies de gramíneas encontradas na área 1 apresentam o ciclo C4 de fixação de carbono, o que lhes confere altas taxas fotossintéticas, em condições de altas temperatura e luminosidade, como ocorre na região Médio Vale do Rio Doce, cuja temperatura média anual é de cerca de $30^{\circ} \mathrm{C}$. Tuffi Santos et al. (2004), trabalhando em áreas de várzea degradadas, verificaram que as famílias mais representativas foram, além de Poaceae, Asteraceae e a sub-família Papilionoideae .

A espécie presente com maior frequência (FRE), na área 1, foi a Acanthospermum australe (Loefl.) Kuntze. No entanto, as espécies encontradas em maior densidade (DEN) foram a Urochloa decumbens (Stapf) R.D.Webster, seguida de Acanthospermum australe e Urochloa plantaginea (Link) R.D.Webster, sendo esta a espécie encontrada em maior abundância (ABN) (Figura 1). Observou-se maior frequência relativa (FRR) para A. australe, seguida por Sidastrum micranthum (A.St.Hil.) Fryxell e Sida carpinifolia L.f. Os resultados mostram maior abundância relativa (DER) para a $B$. decumbens e A. australe. Com relação à abundância relativa (ABR) destacou-se $U$. decumbens e $U$. plantaginea. Com relação ao índice de valor de importância (IVI), que é representado pelo somatório da densidade relativa, da frequência relativa e da dominância relativa - que indica qual espécie tem maior influência dentro de uma comunidade, as espécies mais importantes na área 1 foram: $U$. decumbens, A. australe e $U$. plantaginea seguidas de S. micranthum (Figura 2).

Guimarães et al. (2002), em levantamento fitossociológico de área de pastagem, em Uberlândia, Minas Gerais, verificaram que dentre as espécies exclusivas deste ambiente antropizado, destacam-se Paspalum notatum Flüggé, U. decumbens, Sida rhombifolia L. var. rhombifolia e Emilia sonchifolia (L.) DC. ex Wight. Estas espécies são consideradas, de acordo com Gavilanes \& D'angiere Filho (1991) e por Pedrotti \& Guarim Neto (1998), invasoras de áreas naturais perturbadas e são, também, denominadas de ruderais, comprovando que a alteração da área favorece o seu estabelecimento.

A área 2, Município de Galiléia, apresentou o maior número de espécies entre as oito áreas avaliadas, sendo 28, no total, distribuídas em 11 famílias. As famílias com maior número de espécies foram Fabaceae, Malvaceae e Poaceae com 10, 4 e 4 espécies, respectivamente (Tabela 2). A espécie encontrada com maior frequência e densidade nesta área foi Hyptis suaveolens Poit e, as mais abundantes, H. suaveolens e Ludwigia sericea (Cambess.) H.Hara (Figura 1). H. suaveolens foi também a que mostrou maiores FRR e DER, na área, sendo L. sericea a que apresentou maior ABR. As espécies com maior IVI foram H. suaveolens, Desmodium incanum DC e Sida glaziovii K.Schum (Figura 2). Apesar de a família Fabaceae ter apresentado o maior número de espécies, nesta área, a $H$. suaveolens, uma Lamiaceae, foi a mais importante neste local. Segundo Serrão \& Falesi (1977), o gênero Desmodium é o de maior importância entre as leguminosas encontradas nas pastagens cultivadas, que servem para o pastejo dos animais. Segundo Oliveira et al. (2004), a ocorrência de Desmodium hispidum Franch indica solo deficiente em cálcio.

Na área 3, Município de São Vitor, foram identificadas sete espécies, pertencentes a quatro famílias, sendo a Poaceae e a Malvaceae as famílias com maior número de espécies ocorrentes no local (Tabela 2). Cenchrus ciliaris L. foi a espécie mais frequente e $S$. carpinifolia a que apareceu em maior FRE e ABN, nesta área estudada (Figura 2). As maiores FRR foram observadas para C. cilliaris e $S$. micranthum. Espécies do gênero Cenchrus sp. são comumente encontradas em solos erodidos e compactados (Oliveira et al., 2004). S. carpinifolia também foi a que apresentou maior DER e ABR. Em ordem de importância (IVI), as espécies com maior destaque na área 3 foram $S$. carpinifolia, C. cilliaris e U. decumbens (Figura 3).

As diferenças na intensidade de perturbações no ambiente dessas oito áreas, no caso específico, por pastejo e por pisoteio bovino, seguidas por erosão superficial do solo, podem ter sido responsáveis pelas diferenças na composição florística. Segundo Pandey \& Singh (1991) e 
Tabela 2. Número e diversidade de espécies, número e diversidade de famílias de plantas Daninhas em pastagens degradadas do Médio Vale do Rio Doce em Minas Gerais

\begin{tabular}{|c|c|c|c|c|}
\hline Áreas & $\begin{array}{l}\mathbf{N}^{\circ} \\
\text { espécies }\end{array}$ & Espécies & $\begin{array}{l}\mathrm{N}^{\circ} \\
\text { famílias }\end{array}$ & $\begin{array}{c}\text { Famílias } \\
\left(n^{\circ} \text { espécies }\right)\end{array}$ \\
\hline 1 & 23 & $\begin{array}{l}\text { Urochloa decumbens, Acanthospermum australe, Alysicarpus } \\
\text { vaginalis, Anoda cristata, Arnica montana, Bougainvillea glabra, } \\
\text { Urochloa arecta, Urochloa mutica, Urochloa plantaginea, Cleome } \\
\text { affinis, Cyathula prostrata, Desmodium tortuosum, Digitaria } \\
\text { insularis, Hyptis suaveolens, Lantana fucata, Megathyrsus maximus, } \\
\text { Setaria geniculata, Sida carpinifolia, Sida cordifolia, Sida glaziovii, } \\
\text { Sidastrum micranthum e Stylosanthes viscosa }\end{array}$ & 10 & $\begin{array}{l}\text { Amaranthaceae (1) } \\
\text { Asteraceae (1) } \\
\text { Brassicaceae (1) } \\
\text { Euphorbiaceae (1) } \\
\text { Fabaceae (3) } \\
\text { Lamiaceae (1) } \\
\text { Malvaceae (5) } \\
\text { Nyctaginaceae (1) } \\
\text { Poaceae (8) } \\
\text { Verbenaceae (1) }\end{array}$ \\
\hline 2 & 28 & $\begin{array}{l}\text { Hyptis suaveolens, Desmodium incanum, Sida glaziovii, } \\
\text { Aeschynomene rudis, Urochloa decumbens, Urochloa mutica, } \\
\text { Crotalaria spectabilis, Cyathula prostrata, Desmodium tortuosum, } \\
\text { Desmodium adscendens, Desmodium micranthum, Digitaria } \\
\text { insularis, Jacquemontia heterantha, Lantana fucata, Ludwigia } \\
\text { sericea, Macroptilium atropurpureum, Merremia cissoides, Mi- } \\
\text { mosa malacocentra, Megathyrsus maximus, Pyrostegia venusta, } \\
\text { Remirea maritima, Senna hirsuta, Sida cordifolia, Sida urens, } \\
\text { Sidastrum micranthum, Solanum juripepa, Stylosanthes guianensis, } \\
\text { Tridax procumbens e Vigna unguiculata }\end{array}$ & 11 & $\begin{array}{l}\text { Asteraceae (1) } \\
\text { Bignoniaceae (1) } \\
\text { Convolvulaceae (2) } \\
\text { Cyperaceae (1) } \\
\text { Fabaceae (10) } \\
\text { Lamiaceae (1) } \\
\text { Malvaceae (4) } \\
\text { Onagraceae (1) } \\
\text { Poaceae (4) } \\
\text { Solanaceae (1) } \\
\text { Verbenaceae (1) }\end{array}$ \\
\hline 3 & 7 & $\begin{array}{l}\text { Sida carpinifolia, Cenchrus ciliaris, Acanthospermum australe, } \\
\text { Urochloa decumbens, Jacquemontia heterantha, Lippia Alba, Setaria } \\
\text { vulpiseta e Sidastrum micranthum }\end{array}$ & 4 & $\begin{array}{l}\text { Asteraceae (1) } \\
\text { Convolvulaceae (1) } \\
\text { Malvaceae (2) } \\
\text { Poaceae (3) }\end{array}$ \\
\hline 4 & 16 & $\begin{array}{l}\text { Sida glaziovii, Chloris barbata, Acanthospermum australe, Betonica } \\
\text { officinalis, Urochloa decumbens, Urochloa plantaginea, Desmodium } \\
\text { incanum, Macfadyena unguis-cati, Panicum repens, Paspalum } \\
\text { maritimum, Pyrostegia venusta, Setaria insularis, Sida carpinifolia, } \\
\text { Sida rhombifolia, Sidastrum micranthum, Stylosanthes viscosa }\end{array}$ & 6 & $\begin{array}{l}\text { Asteraceae (1) } \\
\text { Bignoniaceae (2) } \\
\text { Fabaceae (1) } \\
\text { Lamiaceae (1) } \\
\text { Malvaceae (5) } \\
\text { Poaceae (6) }\end{array}$ \\
\hline 5 & 12 & $\begin{array}{l}\text { Urochloa decumbens, Sida glaziovii, Urochloa mutica, Urochloa } \\
\text { plantaginea, Cenchrus ciliaris, Desmodium barbatum, Elephantopus } \\
\text { mollis, Eupatorium maximilianii, Hyparrhenia rufa, Sidastrum } \\
\text { micranthum, Stylosanthes viscosa, Tabebuia avellanedae }\end{array}$ & 5 & $\begin{array}{l}\text { Asteraceae (2) } \\
\text { Fabaceae (2) } \\
\text { Bignoniaceae (1) } \\
\text { Malvaceae (3) } \\
\text { Paoceae (5) }\end{array}$ \\
\hline 6 & 18 & $\begin{array}{l}\text { Urochloa decumbens, Mimosa setosa, Acanthospermum australe, } \\
\text { Urochloa mutica, Urochloa plantaginea, Bromus catharticus, } \\
\text { Croton glandulosus, Desmodium barbatum, Glycine wightii, } \\
\text { Lantana fucata, Melinis minutiflora, Neonotonia wightii, Pyrostegia } \\
\text { venusta, Setaria geniculata, Setaria vulpiseta, Sida glaziovii, } \\
\text { Sidastrum micranthum, Stylosanthes viscosa }\end{array}$ & 8 & $\begin{array}{l}\text { Asteraceae (1) } \\
\text { Bignoniaceae (1) } \\
\text { Euphorbiaceae (1) } \\
\text { Fabaceae (4) } \\
\text { Malvaceae (2) } \\
\text { Mimosoidaceae (1) } \\
\text { Poaceae (7) } \\
\text { Verbenaceae (1) }\end{array}$ \\
\hline 7 & 11 & $\begin{array}{l}\text { Sporobolus indicus, Setaria geniculata, Acanthospermum hispidum, } \\
\text { Aster squamatus, Desmodium incanum, Echinochloa polystachya, } \\
\text { Melinis minutiflora, Schinopsis brasiliensis, Sida carpinifolia, Sida } \\
\text { glaziovii, Stylosanthes viscosa }\end{array}$ & 5 & $\begin{array}{l}\text { Anacardiaceae (1) } \\
\text { Asteraceae (2) } \\
\text { Fabaceae (2) } \\
\text { Malvaceae (2) } \\
\text { Poaceae (4) }\end{array}$ \\
\hline 8 & 6 & $\begin{array}{l}\text { Urochloa decumbens, Sida carpinifolia, Lantana fucata, Sida } \\
\text { glaziovii, Sidastrum micranthum, Solanum lycocarpum }\end{array}$ & 4 & $\begin{array}{l}\text { Malvaceae (3) } \\
\text { Poaceae (1) } \\
\text { Solanaceae (1) } \\
\text { Verbenaceae (1) }\end{array}$ \\
\hline Total & 68 & & & 17 \\
\hline
\end{tabular}



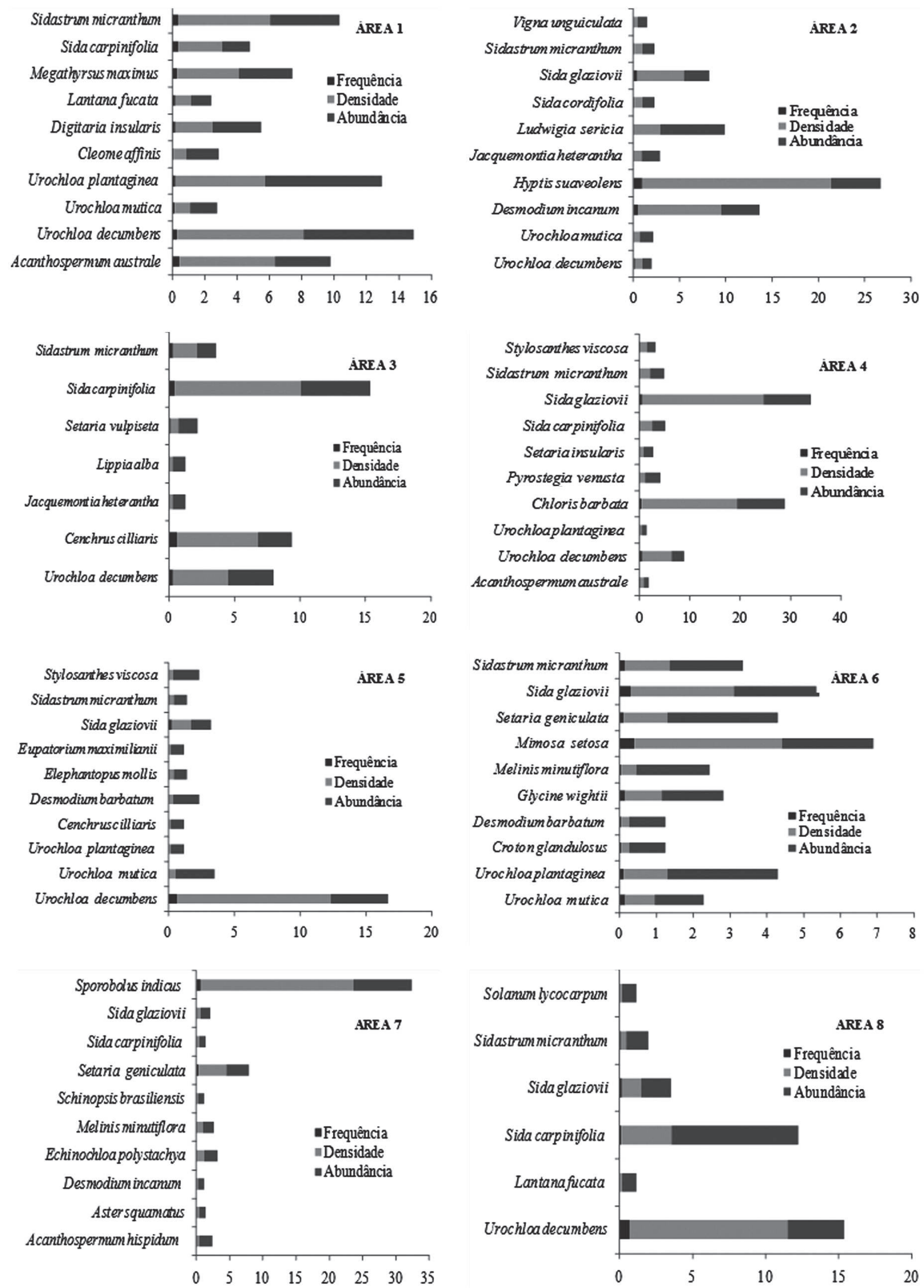

Figura 1. Frequência, densidade e abundância de espécies de plantas daninhas em oito áreas de pastagens da Região do Médio Vale do Rio Doce, Minas Gerais.

Rev. Ceres, Viçosa, v. 61, n.4, p. 502-510, jul/ago, 2014 

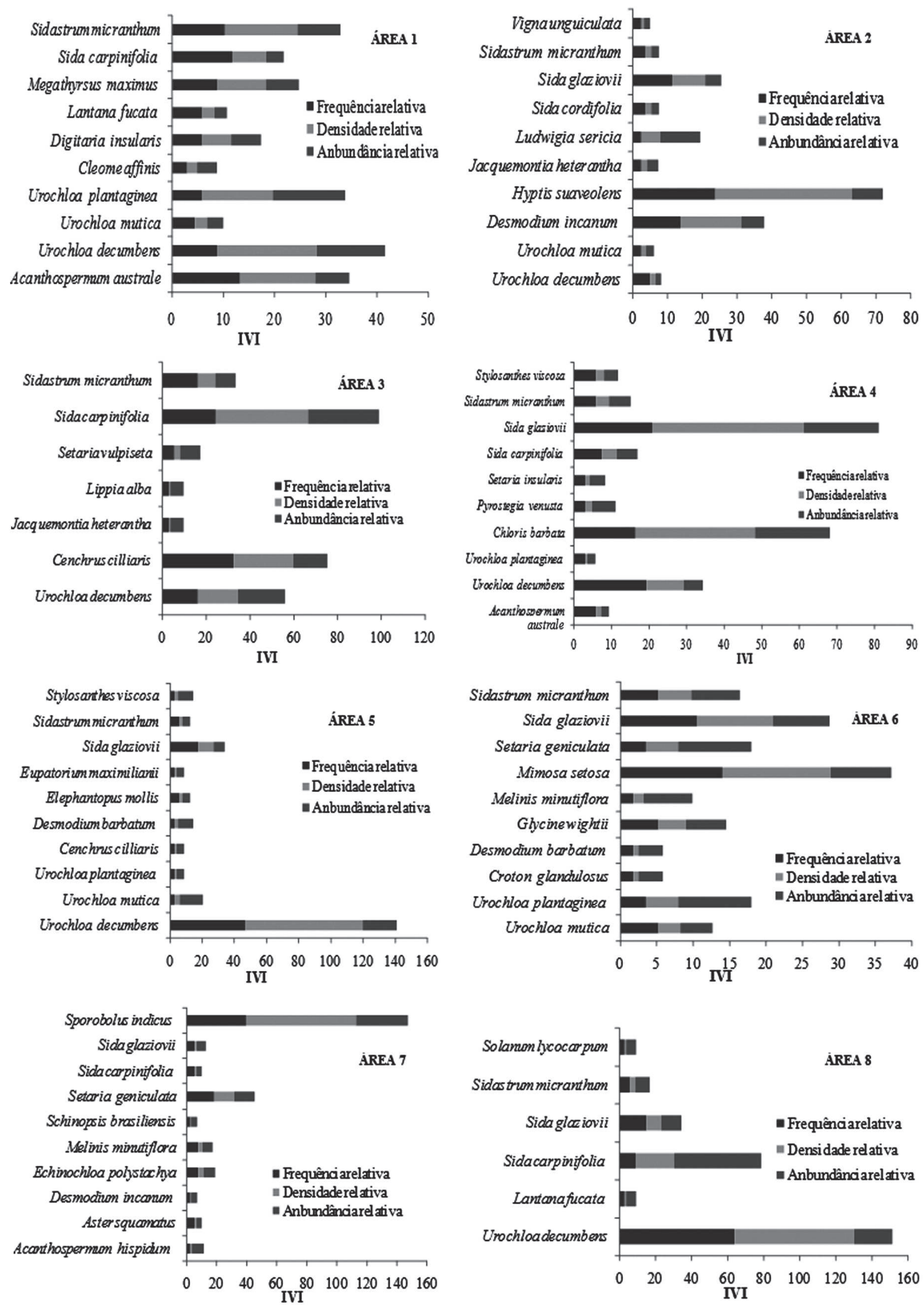

Figura 2. Frequência relativa, densidade relativa, abundância relativa e índice de valor de importância de espécies daninhas em oito áreas de pastagens da Região do Médio Vale do Rio Doce, Minas Gerais. 
Belsky (1992), as áreas protegidas tendem a apresentar menor riqueza de espécies que áreas pastejadas correspondentes, mas esta alteração está diretamente relacionada com a intensidade do pastejo. Para os autores, a riqueza de espécies aumenta sistemas de produção conduzidos com pastejo moderado e declina com pastejo intenso. Segundo os mesmos, a maior cobertura vegetal em áreas preservadas, em relação às pastejadas, ocorre por causa da presença de espécies dominantes.

Identificaram-se, na área 4, Município de Engenheiro Caldas, distrito de Divino do Traira, 16 espécies, distribuídas em seis famílias. As famílias com maior número de espécies foram Poaceae (6) e Malvaceae (5) (Tabela 2). As espécies mais frequentes foram $U$. decumbens, $S$. glaziovii e Chloris barbata (L.) Sw , sendo que estas últimas apresentaram, também, maior DEN e ABN. S. glaziovii também foi a que apresentou maiores FRR, DER e ABR, sendo a mais importante na área, apresentando maior IVI. Com relação a esta variável, pode-se destacar também as espécies $C$. barbata e $U$. decumbens (Figura 1).

$\mathrm{Na}$ área 5, Município de Governador Valadares, distrito de Baguarí, foram encontradas 12 espécies, pertencentes a cinco famílias, sendo Poaceae e Malvaceae as famílias com maior número de espécies (Tabela 2). A $U$. decumbens apresentou-se com maior frequência, densi- dade e abundância, na área 5, com maior FRR, DER, ABR e IVI, sendo, desta forma, a mais importante da área (Figura 2).

Observaram-se, na área 6, Município de Engenheiro Caldas, oito famílias, representadas por 18 espécies. As famílias com maior número de espécies foram Poaceae e Fabaceae, com sete e quatro espécies, respectivamente (Tabela 2). As espécies mais frequentes e que ocorreram em maior densidade nesta área foram: Mimosa setosa Benth e S. glaziovii, Setaria geniculata (Lam.) P.Beauv, $U$. plantaginea, M. setosa e $S$. glaziovii foram as mais abundantes (Figura 1). M. setosa e S. glaziovii também apresentaram maior FRR e DER. Já a U. plantaginea apresentou maior ABR. M. setosa e S. glaziovii foram consideradas as espécies mais importantes da área (Figura 2).

No Município de Governador Valadares, distrito de Baguarí, área 7, foram identificadas cinco famílias, representadas por 11 espécies, com destaque para as Poaceae e Asteraceae, com quatro e duas espécies, respectivamente (Tabela 2). Nessa área, assim como foi observado na área 5, houve forte dominância de Sporobolus indicus (L.) R.Br, com maiores valores de FRE, DEN, ABN, FRR, DER, ABR e IVI (Figuras 1 e 2).

Os resultados mostram, na oitava área, Município de Governador Valadares, distrito de Marcos Bretas, a iden-

Índice de similaridade (\%)

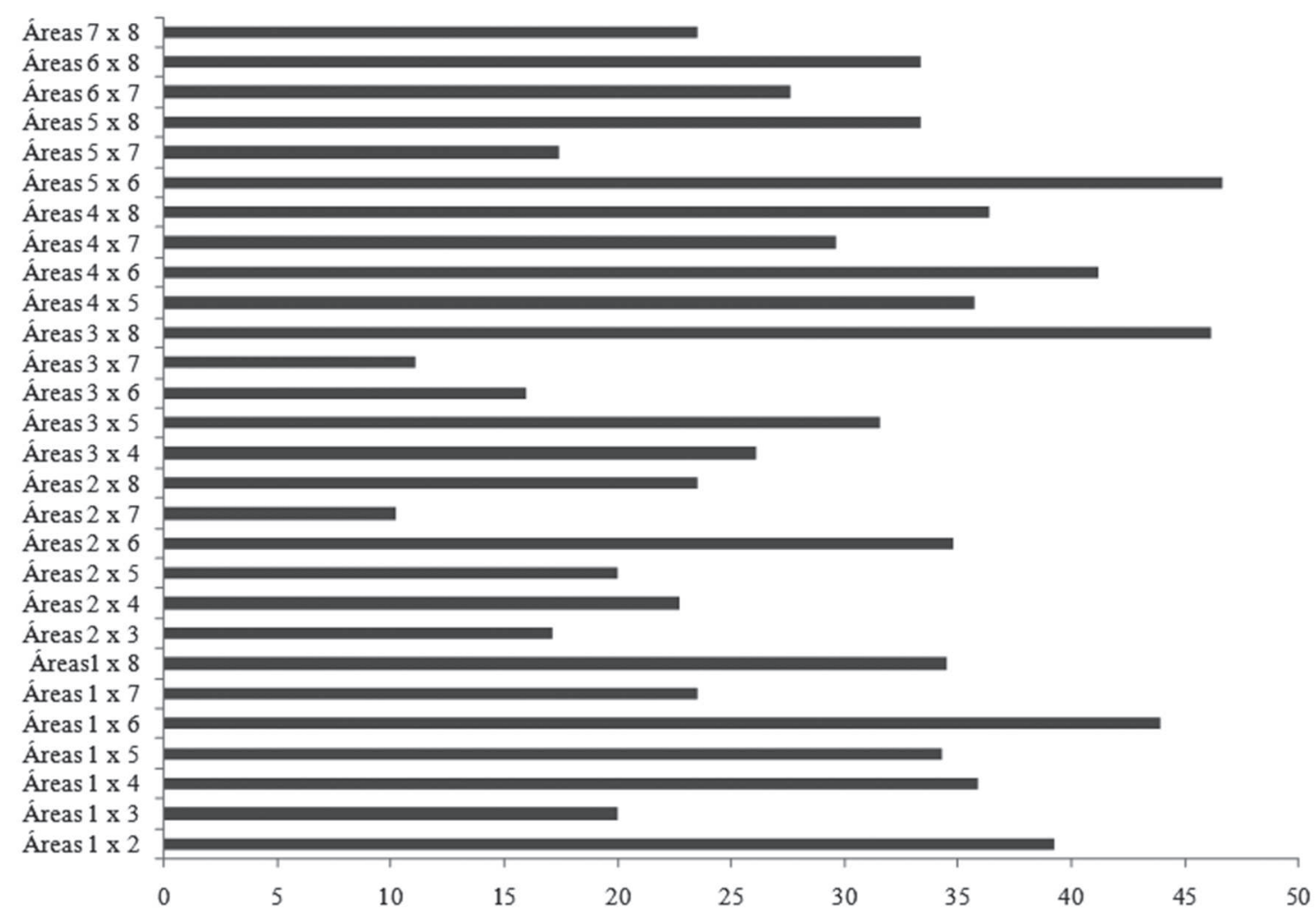

Figura 3. Índice de similaridade (\%) entre as oito áreas de pastagens avaliadas quanto à incidência de plantas daninhas na Região do Médio Vale do Rio Doce, Minas Gerais.

Rev. Ceres, Viçosa, v. 61, n.4, p. 502-510, jul/ago, 2014 
tificação de apenas sete espécies, distribuídas em quatro famílias, sendo Malvaceae a com maior número de espécies (Tabela 2). Nessa área, $U$. decumbens apresentou maior FRE, FRR, DEN e DER e, S. carpinifolia, maiores ABN e ABR (Figuras 1 e 2). Santana (2009), trabalhando em áreas de pastagens, com solos altamente degradados, na Região de Gurupi, Tocantins, constatou que as principais plantas invasoras encontradas foram: $H$. suaveolens, $S$. obtusifolia, S. cordifolia, S. micranthum, e Waltheria indica $\mathrm{L}$, inferindo que espécies da família Malvaceae podem indicar grau de degradação e compactação elevados.

Foram identificadas 68 espécies, nas oito áreas avaliadas (Tabela 2), destacando-se $U$. decumbens, $S$. glaziovii e $S$. carpinifolia. Verificaram-se espécies do gênero Sida em todas as áreas avaliadas, apresentando alto IVI, isto é, essas espécies foram classificadas entre as dez mais importantes em suas respectivas áreas. Segundo Oliveira et al. (2004), a S. carpinifolia é uma espécie bioindicadora de impacto em ambientes agrícolas, sendo que sua presença é comum em solos erodidos e solos compactados.

Entre as áreas, constatou-se grande variação na diversidade, sendo a maior riqueza observada na área 2 , município de Galiléia, que apresentou 28 espécies. A menor diversidade foi observada na área 8, pertencente ao distrito de Marcos Bretas, Governador Valadares, com apenas seis espécies, destacando-se $S$. carpinifolia como a mais abundante (Figura 1). Essa variação espacial da dominação das plantas infestantes, observada neste estudo, pode ser reflexo de diferentes sistemas de manejo do solo e diferentes práticas culturais, empregados pelos agricultores em cada local, pois esses fatores influenciam a dinâmica das populações de plantas daninhas (Oryokot et al., 1997). A introdução de Megathyrsus maximus (Jacq.) B. K.Simon \& S.W. L. Jacobs, de maneira generalizada, na região (Baruqui, 1982), apesar de constituir fator fundamental para caracterizar a pecuária como importante segmento na capitalização dos produtores rurais, não apresentou sustentabilidade. Esse fato é evidenciado pelo baixo valor de importância dessa espécie, aparecendo, com mínima importância, em somente duas das oito áreas avaliadas.

Quanto à similaridade entre as áreas, o maior valor foi observado entre as áreas 5 e 6 dos municípios de Governador Valadares, distritos de Baguarí, e Engenheiro Caldas (IS de aproximadamente 47\%). Entre as demais áreas, em média, os valores de similaridade não ultrapassaram $28,5 \%$, indicando baixa homogeneidade entre elas, podendo, isso, ser explicado pelo fato de serem locais distintos, quanto à distância geográfica, e com condições específicas de solo. A menor similaridade, observada entre as áreas 3 e 7, dos municípios de São Vitor e de Governador Valadares, distrito de Baguarí, relaciona-se ao fato de com- partilharem somente uma espécie em comum, resultando em IS de aproximadamente 11\% (Figura 4).

Os baixos valores de IS e a diversidade de espécies com alto IV, nas áreas avaliadas, sugerem que sejam adotados manejos diferentes, para cada local, visto que o uso de um único herbicida para controle destas plantas daninhas será pouco eficiente. Esses resultados corroboram os encontrados por Curtinhas et al. (2010), que, também, relataram baixa similaridade entre cinco áreas representativas das microbacias que compõem a rede hidrográfica do Médio Rio Doce e, ainda, evidenciaram heterogeneidade de espécies herbáceas e podem ser consequências da integração de fatores, como distância geográfica, altitude e manejo anterior, adotado em cada área.

\section{CONCLUSÕES}

As áreas estudadas apresentaram grande variação na diversidade de plantas daninhas.

Ao todo foram identificadas 68 espécies, sendo que o gênero Sida foi encontrado em todas as áreas.

As espécies $U$. decumbens, $S$. glaziovii e $S$. carpinifolia foram as plantas daninhas com maior ocorrência em pastagens degradadas, no Médio Vale do Rio Doce - MG, e merecem atenção especial no manejo de recuperação dessas áreas.

\section{REFERÊNCIAS}

Baruqui FM (1982) Inter-relações solo-pastagens nas regiões mata e rio doce do estado de minas gerais. Dissertação Mestrado. Universidade Federal de Viçosa, Viçosa. 119p.

Belsky AJ (1992) Effects of grazing, competition, disturbance and fire on species composition and diversity in grassland communities. Journal Vegetation Science, 3:187-200.

Curtinhas JN, Santos J B, Vicente N M F \& Perez A L (2010) Caracterização fitossociológica da vegetação herbácea de áreas alteradas pela atividade agropecuária na região do Médio Vale do Rio Doce, Minas Gerais. Revista Ceres, 57:321-329.

Ellingson LJ, Kauffman JB, Cummings DL, Sanford RL \& Jaramillo VJ (2000) Soil N dynamics associated with deforestation, biomass burning, and pasture conversion in a Mexical tropical dry forest. Forest Ecology and Management, 137:41-51.

Fávero C, Lovo IC \& Mendonça E de S (2008) Recuperação de área degradada com sistema agroflorestal no Vale do Rio Doce, Minas Gerais. Revista Árvore, 32:861-868.

Gavilanes ML \& D'angieri Filho CN (1991) Flora ruderal da cidade de Lavras, MG. Acta Botanica Brasilica, 5:77-88.

Gomes GLGC, Ibrahim FN, Macedo GL, Nobrega LP \& Alves E (2010) Cadastramento fitossociológico de plantas daninhas na bananicultura. Planta daninha, 28:61-68.

Guimarães AJM, Araújo GM \& Corrêa GF (2002) Estrutura fitossociológica em área natural e antropizada de uma vereda em Uberlândia, MG. Acta Botanica Brasilica, 16:317-329. 
Lara JFR, Macedo JF \& Brandão M (2003) Plantas daninhas em pastagens de várzeas do estado de Minas Gerais. Planta Daninha, 21:11-20.

Kauffman JB, Cummings DL \& Ward DE (1998) Fire in the Brazilian Amazon: 2- biomass, nutrient pools and losses in cattle pasture. Oecologia, 113:415-427.

Mueller-Dombois \& D Ellenberg HA (1974) Aims and methods of vegetation ecology. New York, John Wiley. 547p.

Oliveira FNS, Freire FCH \& Aquino ARL (2004) Bioindicadores de impacto ambiental em sistemas agrícolas orgânicos. Disponível em: < http://www.cnpat.embrapa.br/cnpat/cd/jss/acervo/ Dc_093.pdf>. Acessado em: 14 de abril de 2013.

Oryokot JOE, Murphy SD \& Swanton CJ (1997) Effect of tillage and corn on pigweed (Amaranthus spp.) seedling emergence and density. Weed Science, 45:120-126.

Pandey CB \& Singh JS (1991) Influence of grazing and soil conditions on secondary savanna vegetation in India. Journal Vegetation Science, 2:95-102

Pedrotti DE \& Guarim Neto G (1998) Flora Ruderal da Cidade de Cuiabá, Mato Grosso, Brasil. Acta Botanica Brasilica, 12:135143 .

Peron AJ \& Evangelista A R (2004) Degradação de pastagens em regiões de cerrado. Ciência e Agrotecnologia, 28:655-661.

Pitelli RA (2000a) Estudos fitossociológicos em comunidades infestantes de agroecossistemas. Journal Conserb, 1:1-7.

Pitelli RA (2000b) Estudo fitossociológico de uma comunidade infestante da cultura da cebola. Journal Conserb, 1:1-6.
Post BJ Multivariate analysis in weed science (1998). Weed Research, 28:425-30.

Santana SC (2009) Indicadores físicos da qualidade de solos no monitoramento de pastagens degradadas na região sul do Tocantins. Tese de Doutorado. Universidade Federal de Tocantins, Gurupi. 76p.

Serrão EAS \& Falesi IC (1977) Pastagens do trópico úmido brasileiro. In: $4^{\circ}$ Simpósio Sobre Manejo de Pastagens, Piracicaba. Escola Superior de Agricultura "Luiz de Queiroz". CD ROM.

Silva AA, Werlang RC \& Ferreira LR (2002) Controle de plantas daninhas em pastagens. In: $1^{\circ}$ Simpósio sobre manejo estratégico da pastagem, Viçosa. Anais, SBZ. p.273-310.

Silva DSM \& Dias Filho MB (2001) Banco de sementes de plantas daninhas em solo cultivado com pastagens de Brachiaria brizantha e Brachiaria humidicola de diferentes idades. Planta Daninha, 19:179-185.

Silva RM, Yamashita OM, Carvalho MAC, Meurer L, Realto GB, Ribas C, Franceschet VL, Silva JAN, Oliveira MA, Pereira RA, Silva CJ \& Silva CAH (2013) Levantamento fitossociológico de plantas daninhas em pastagem degradada submetida a diferentes sistemas de recuperação. Cultivando o Saber, 6:152-161.

Sorensen TA (1972) method of stablishing groups of equal amplitude in plant society based on similarity of species content. In: Odum EP (Ed.) Ecologia. $3^{\mathrm{a}}$ ed. México, Interamericana. 640p.

Tuffi Santos LD, Santos IC, Oliveira CH, Santos MV, Ferreira FA \& Queiroz DS (2004) Levantamento fitossocilógico em pastagens degradadas sob condições de várzeas. Planta Daninha, 22:343-349 